\title{
Imaging of pancreas transplantation and its complications
}

\author{
Manuela França • Manuela Certo • Lasalete Martins • Pedro Varzim • \\ Manuel Teixeira • António Castro Henriques • António Manuel Ribeiro • \\ Filipe Caseiro Alves
}

Received: 25 June 2010 / Accepted: 10 September 2010 /Published online: 1 October 2010

(C) European Society of Radiology 2010

\begin{abstract}
Pancreas transplantation is an effective treatment for type 1 diabetes mellitus and is being increasingly performed worldwide. Early recognition of graft-related complications is fundamental for graft survival; thus, radiologists must be aware of the transplantation technique, pancreas-graft imaging and postoperative complications. We present normal pancreas-graft imaging appearances and the imaging features of postoperative complications.
\end{abstract}

Keywords Diabetes mellitus · Type $1 \cdot$ Pancreas transplantation $\cdot$ Pancreas-kidney $\cdot$ Complications

\footnotetext{
M. França $(\bowtie)$

R Vitorino Nemesio, 162,

4470-221 Maia, Portugal

e-mail: mariamanuela.franca@gmail.com

M. França $\cdot$ M. Certo $\cdot$ P. Varzim • A. M. Ribeiro

Radiology Department, Centro Hospitalar do Porto,

Largo Prof. Abel Salazar,

4099-001 Porto, Portugal

L. Martins $\cdot$ A. C. Henriques

Nephrology Department, Centro Hospitalar do Porto,

Largo Prof. Abel Salazar,

4099-001 Porto, Portugal

M. Teixeira

Surgery Department, Centro Hospitalar do Porto,

Largo Prof. Abel Salazar,

4099-001 Porto, Portugal

F. C. Alves

Radiology Department, Coimbra University Hospital, Praceta Mota Pinto/Av. Bissaya Barreto,

Coimbra, Portugal
}

\section{Introduction}

Pancreatic transplantation is currently the only effective treatment for type 1 diabetes mellitus, allowing long-term glycaemic control without exogenous insulin injections. In most cases, it is performed as a simultaneous pancreaskidney (SPK) transplantation from the same donor but it can be performed after kidney transplantation or, rarely, as an isolated transplantation [1]. The first pancreatic transplantation was performed in 1966 [2] and, since then, different procedures and immunosuppressive regimens have been developed in order to improve graft survival rates. At our institution, one of the first referral transplant centres in Portugal, more than 100 pancreas transplants have been performed since 2000. Most of these (96\%) were SPK transplants from the same donor. The 9-year mean pancreatic graft survival rate was $78 \%$ at our institution.

Early recognition of graft-related complications is fundamental for graft survival, and radiologists must be aware of the transplantation technique, pancreas-graft imaging and postoperative complications. In this article, we present our 10-year experience with the procedure, describing the normal postoperative imaging findings and complications in a large series of 104 patients.

\section{Transplantation procedure}

At our centre, transplantation of the whole pancreatic graft is performed with a duodenal segment, with systemic endocrine drainage via the grafted portal vein into the recipient's inferior vena cava or common iliac vein, and enteric exocrine drainage via the anastomosis of the donor's duodenal segment to the recipient's small bowel (Fig. 1). Pancreatic endocrine drainage may also be performed to the 


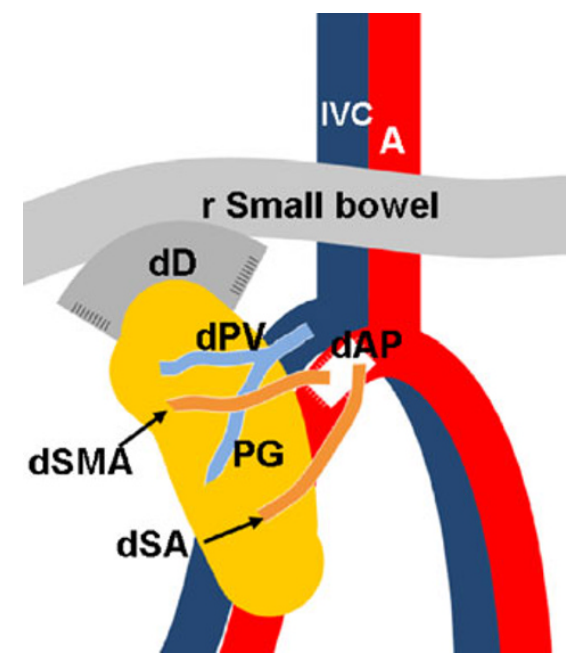

Fig. 1 Illustration of the pancreas transplantation technique performed at our institution, using duodeno-enterostomy for exocrine drainage and systemic endocrine drainage. The donor's aortic patch $(d A P)$ with the origin of the superior mesenteric artery $(d S M A)$ and the splenic artery $(d S A)$ is anastomosed to the recipient's common iliac artery; the donor's portal vein $(d P V)$ is anastomosed to the recipient's common iliac vein. $P G$ pancreatic graft, $d D$ donor's duodenum, $r$ Small bowel recipient small bowel, $I V C$ inferior vena cava, $A$ aorta

recipient's portal venous system $[3,4]$ and/or the exocrine drainage may be derived to the bladder, but these surgical techniques have never been performed at our institution. Arterial supply to the pancreatic graft is performed through a donor's aortic patch, containing the splenic artery and the superior mesenteric artery (SMA), which is anastomosed to the recipient's common or external iliac artery. The pancreatic graft is placed intraperitoneally laterally in the pelvis (preferably on the right side), with the duodenal segment facing cephalad. The donor's duodenal segment is anastomosed side-to-side to the recipient's small bowel (duodeno-enterostomy). The recipient's native pancreas in the upper abdomen is left untouched. When simultaneous kidney-pancreas transplantation is performed, the donor's kidney is preferably placed in the contralateral side of the pelvis, most frequently at the left iliac fossa.

\section{Imaging of the transplanted pancreas}

Imaging evaluation of the pancreas transplant grafts is commonly performed by a multi-technique approach. Ultrasound is usually the first technique to be used to search for early complications [4], as it is routinely performed in the postoperative period (in the first $24 \mathrm{~h}$ ). In grey-scale B-mode, the normal pancreatic graft presents homogeneous echotexture, lower than the native pancreas and the surrounding mesenteric or epiploic fatty tissue. Doppler imaging provides vascular assessment (Fig. 2), with allograft vein velocities ranging between 10 and
$60 \mathrm{~cm} / \mathrm{s}$. In the immediate postoperative period, arterial velocities may be as high as $400 \mathrm{~cm} / \mathrm{s}$ at the anastomotic site, due to kinking or oedema of the anastomosis, but are usually reduced at follow-up examinations. The resistive index (RI) may be as high as 0.9 and be variable throughout the gland, with even higher values at the tail segment. This variability makes it of limited value for the diagnosis of graft rejection [4-6].

Contrast-enhanced computed tomography (CT) allows excellent evaluation of the graft's parenchyma, vascular and enteric anastomosis, and detects several postoperative complications, such as ascites, fluid collections, pneumoperitoneum or vascular thrombosis $[7,8]$. CT is generally required after an abnormal ultrasound or whenever the patient presents unexplained fever, abdominal pain or when abnormal laboratory data are found. Multidetector (MD) $\mathrm{CT}$ allows multiplanar imaging and three-dimensional (3D) reconstructions of the graft's vascular anatomy to better advantage (Figs. 3 and 4). As with imaging the normal pancreas, the graft should display homogeneous enhancement, with the main pancreatic duct (MPD) being unrecognisable or showing a minor degree of dilatation.

Magnetic resonance imaging (MRI) has the advantage of allowing exploitation of the native signal intensity of the graft besides the vascular information provided by Gdenhanced dynamic study (Fig. 5). Also, magnetic resonance cholangiopancreatography (MRCP) helps to depict ductal abnormalities [8]. At our institution, MRI is rarely used for examination of pancreatic graft-related complications because of its lower spatial resolution, creating difficulties in the assessment of the enteric anastomosis, and, also, because of technical constraints involved in imaging acutely ill and intensively monitored patients. Normal pancreas should be isointense to the renal graft parenchyma on T1-weighted images, with an intermediate signal on T2weighted images and homogeneous enhancement after intravenous contrast medium administration [5,9].

The role of digital subtraction angiography is reserved for cases where vascular abnormalities need to be confirmed or when endovascular therapy is sought [8].

\section{Complications of pancreatic transplantation}

In the immediate postoperative period, one should expect to find small peri-graft fluid collections, donor's duodenal wall thickening, mild pancreatic duct dilation, slight stranding of peri-pancreatic fat or oedematous swelling of the donor's remaining mesenteric fat, surrounding the mesenteric artery (Fig. 6) [5]. These imaging findings are usually self-limited, normally seen to be resolving spontaneously on follow-up examinations. Post-transplantation complications of the allograft may be classified as 
Fig. 2 Normal findings at colour and spectral Doppler ultrasound examination of pancreatic graft veins (a) and arteries (b) displaying good organ perfusion and clear arterial spectra with normal resistive index
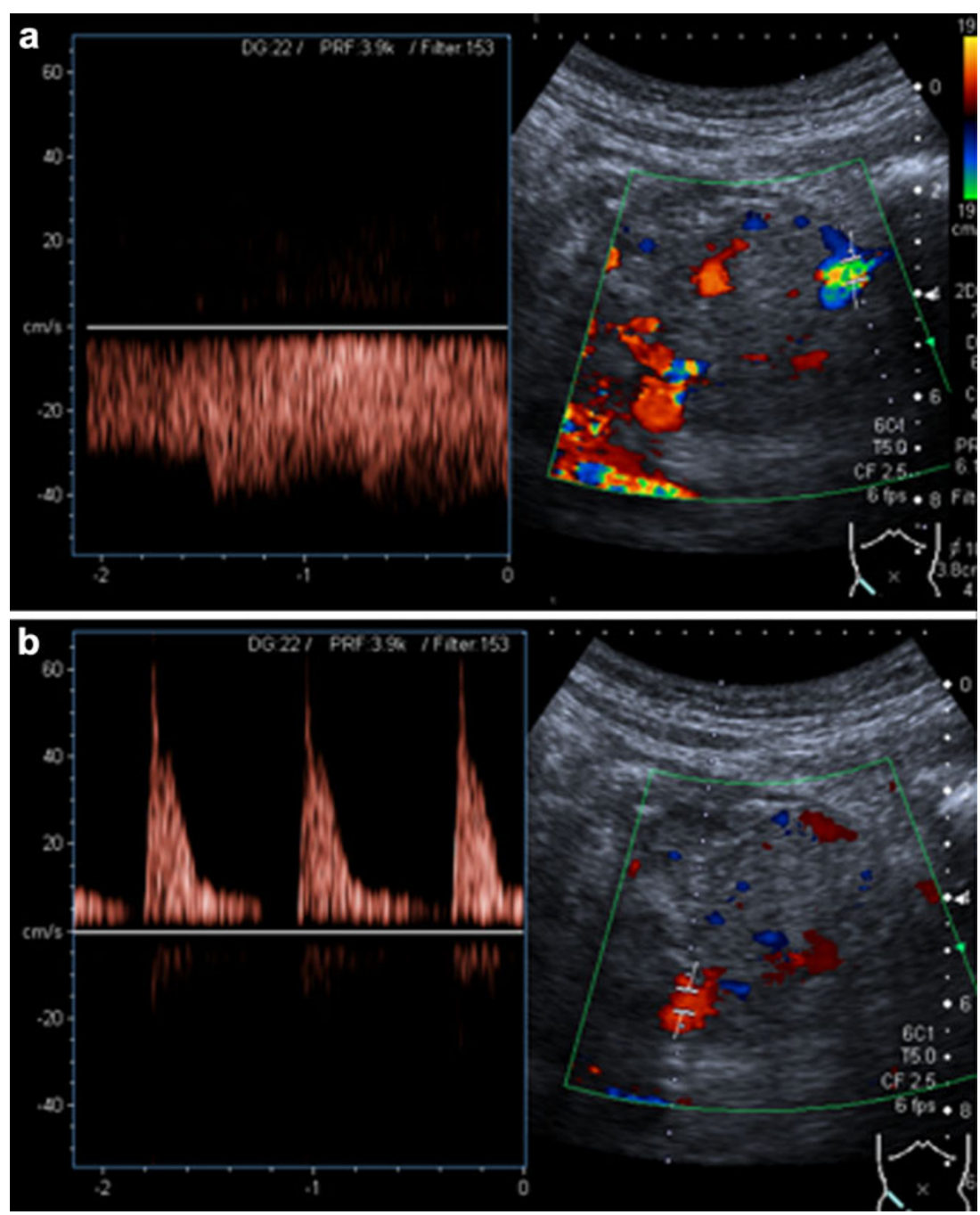

parenchymal, infectious, enteric or vascular. In our series, the most frequent complications, by order of relevance, were graft pancreatitis, infection, and necrosis secondary to arterial or venous thrombosis. Less common complications included pancreatic fistula, bleeding, duodenal anastomosis dehiscence and small bowel obstruction (Table 1).

Allograft parenchymal complications

\section{Acute pancreatitis}

Graft acute pancreatitis, mild and self-limited, is frequently seen in the early postoperative period and is due to reperfusion injury [8]. Severe pancreatitis is uncommon,

Fig. 3 MPR coronal oblique contrast-enhanced MDCT images showing a normal pancreatic graft arterial supply after SPK transplantation. The donor's superior mesenteric artery (SMA) supplies the pancreatic graft head (white asterisk), and the donor's splenic artery irrigates the graft body and tail (black asterisk)

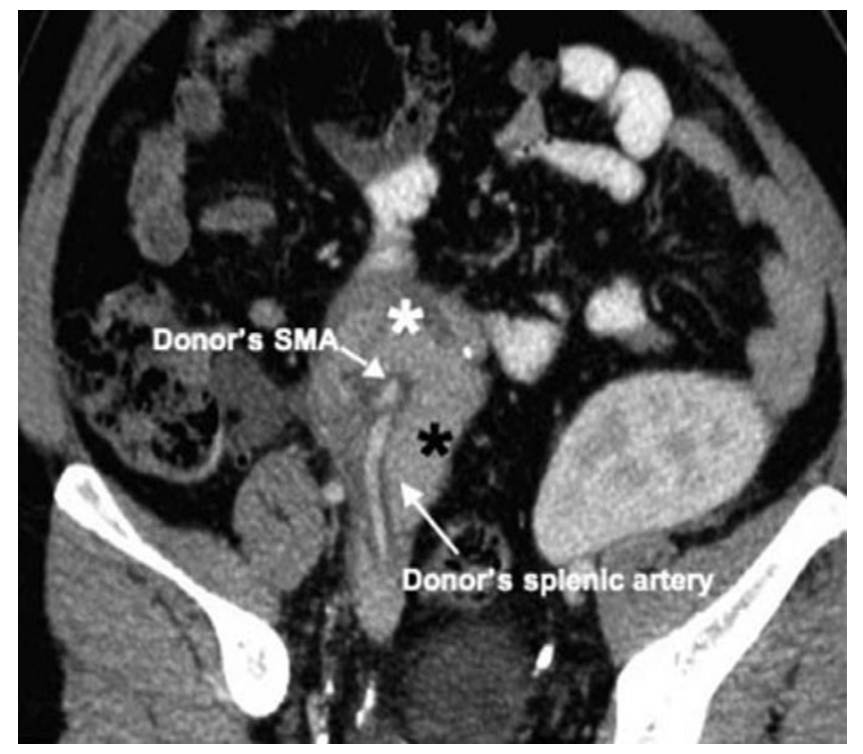




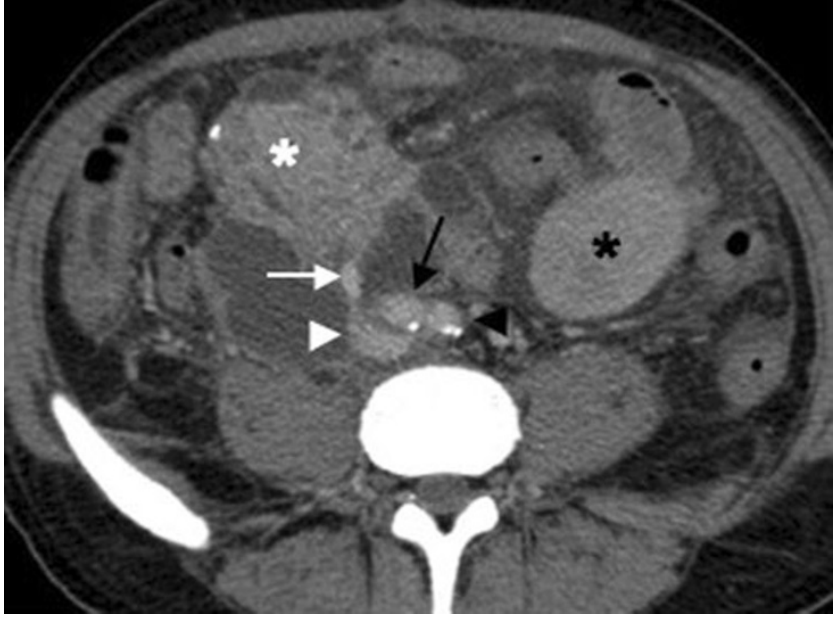

Fig. 4 Axial contrast-enhanced MDCT after SPK transplantation. End-to-side anastomosis is used to connect the donor's portal vein (white arrow) to the recipient's right common iliac vein (white arrowhead). Homogeneous fluid collections adjacent to the pancreatic graft are observed. Black arrow right common iliac artery; black arrowhead left common iliac artery; white asterisk pancreatic graft; black asterisk renal graft

occurring in about $10 \%$ of allografts [5]. Imaging findings are non-specific consisting of normal or enlarged pancreatic allograft, showing heterogeneous contrast enhancement, adjacent fat stranding and fluid collections (Fig. 7). Necrotising pancreatitis may occur in about $2-4 \%$ of the allografts [5] and is the most severe form of acute pancreatitis. Necrosis can result either from pancreatitis itself or from direct vascular occlusion. On ultrasound, necrosis manifests as hypoechoic areas within the graft parenchyma, sometimes with hyperechoic foci suggesting gas formation. Doppler interrogation assists in confirming absent arterial or venous flow within the affected segments of the pancreatic graft [9]. Contrast-enhanced CT or MRI is

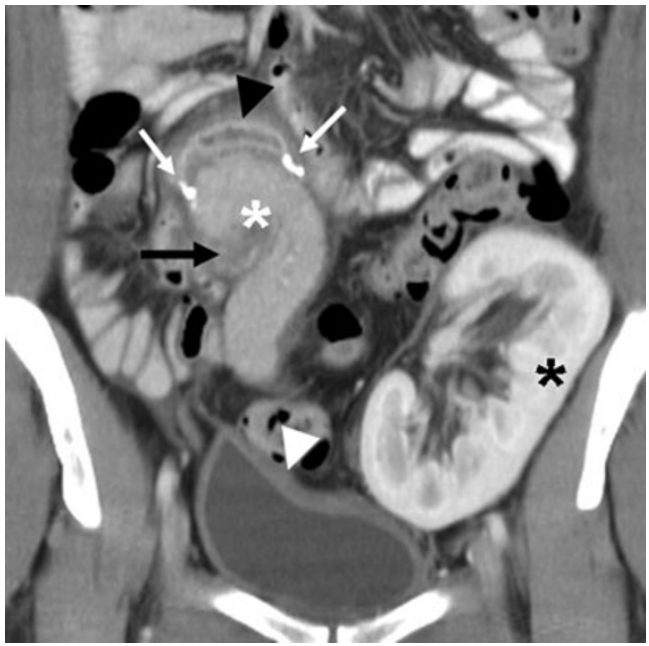

Fig. 6 Coronal reformatted MDCT image shows homogeneous enhancement of the pancreatic (white asterisk) and renal grafts (black asterisk) after SPK transplantation. The pancreas is placed laterally in the pelvis, on the right side, with the attached donor's duodenal segment facing cephalad (black arrowhead), which anastomoses to the recipient's jejunum. Surgical staples are present at the extremities of the duodenal segment (white arrows). Note the oedematous swelling of the donor's remaining mesenteric fat (black arrow) surrounding the pancreatic graft vessels. These findings are to be expected in the early postoperative period

exquisitely sensitive to diagnosing and determining the extent of parenchymal necrosis due to lack of enhancement and possible gas formation (Fig. 8) [8].

\section{Pseudocyst formation}

Pseudocysts typically develop in the severe form of pancreatitis, usually being located within (Fig. 9) or adjacent to the graft (Fig. 10) [5, 8]. Imaging reveals thinwalled fluid collections. Wall thickening showing contrast
Fig. 5 A 32-year-old female patient after SPK transplantation. a Coronal MIP image in the arterial phase of an MRA study demonstrates the arterial vessel anatomy of the pancreatic graft. Note that the donor's SMA (arrow) presents normal calibre and the donor's splenic artery (arrowhead) shows a stenosis with mild post-stenotic dilation. $\mathbf{b}$ MR angiography in the arterial phase shows normal enhancement of the pancreas (arrow) and kidney (arrowhead) transplants. At the lower pole of the kidney graft a large lymphocele is seen (asterisk)
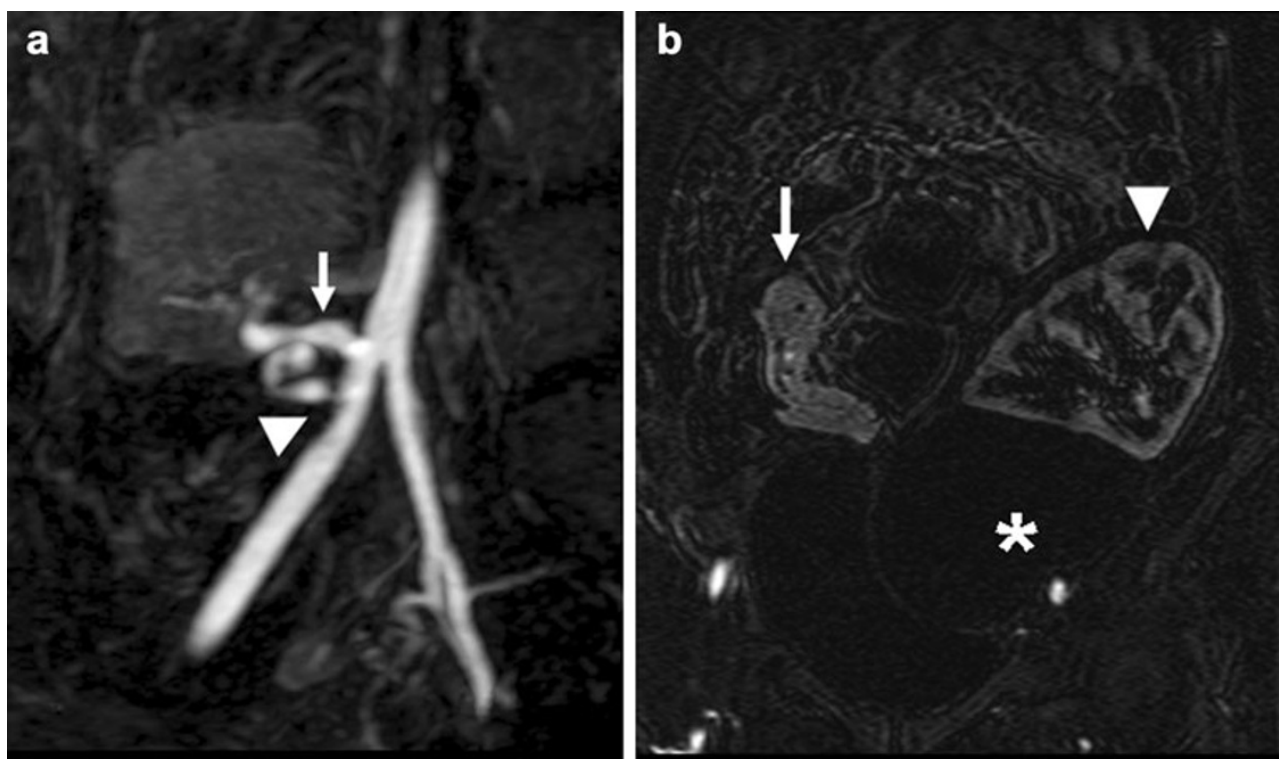
Table 1 Complications of pancreatic transplantation

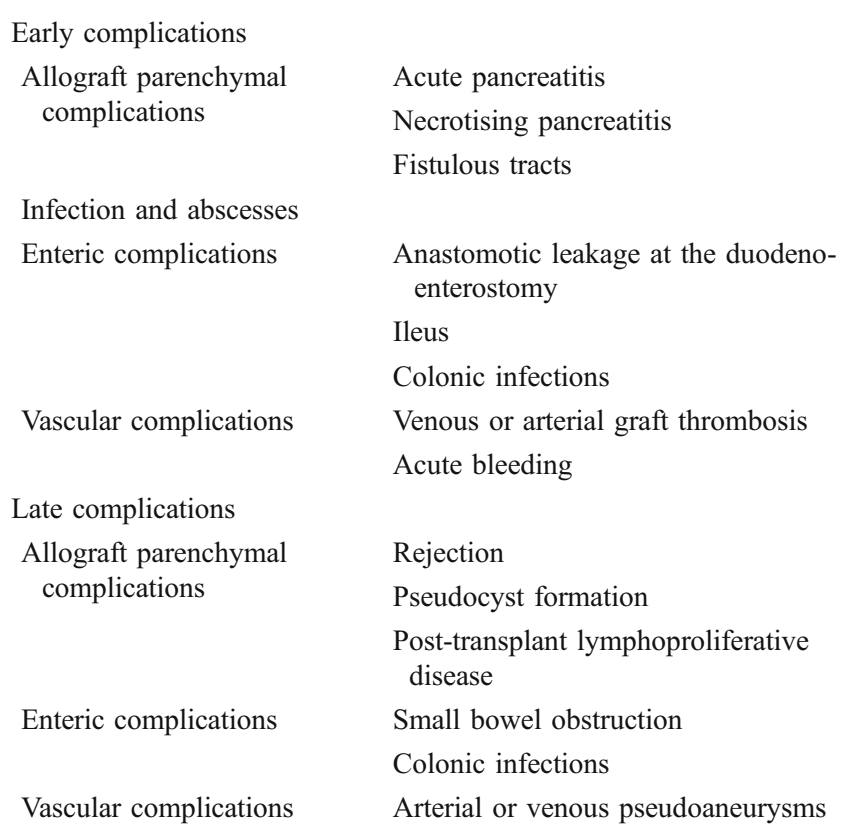

enhancement and heterogeneous content should raise the suspicion of super-seeded infection, which may be managed by percutaneous drainage.

\section{Rejection}

Rejection is less common in pancreatic transplantation than in renal transplantation but it is a common cause of pancreatic graft loss [5]. Imaging findings are non-specific and they may look similar to other complications, such as pancreatitis. Contrast-enhanced CT or MRI may show
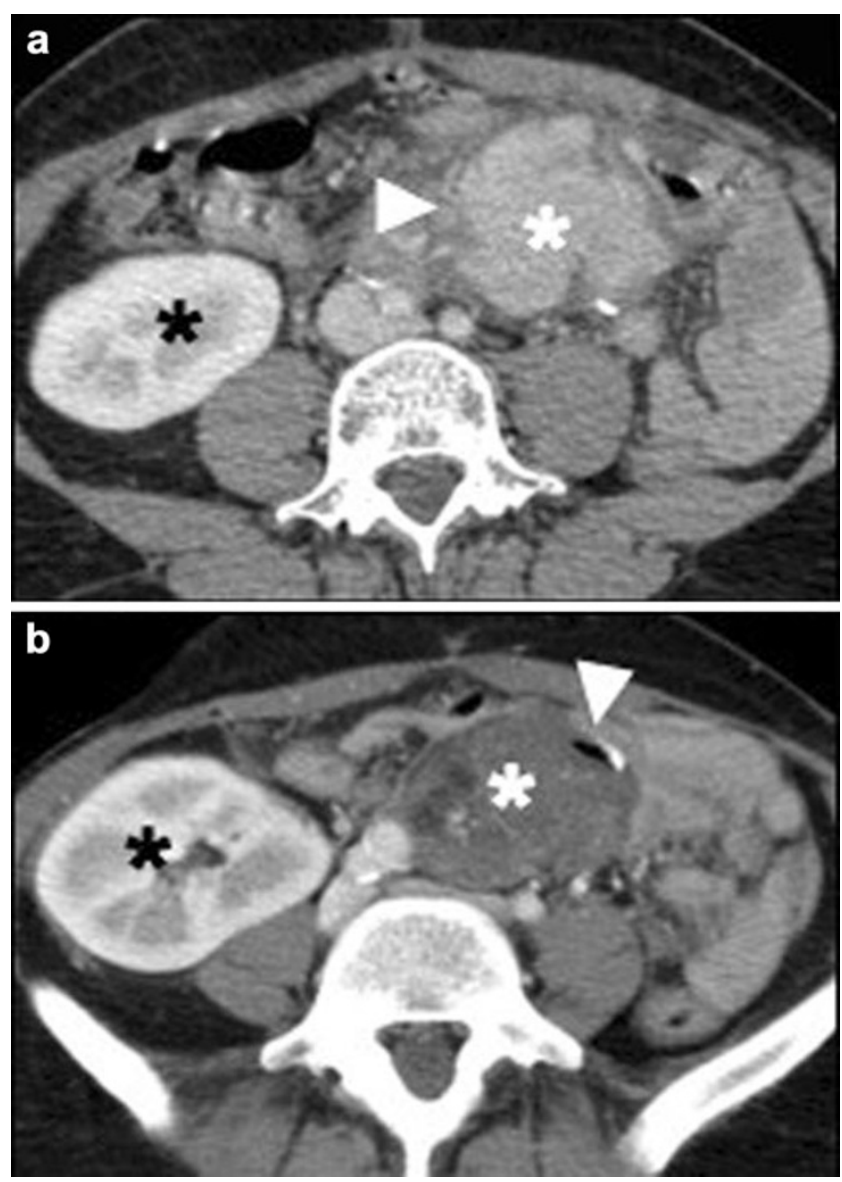

Fig. 8 A 40-year-old female patient after SPK transplantation. Axial contrast-enhanced MDCT (a) shows an enlarged pancreatic graft (white asterisk), with homogeneous parenchymal enhancement and peri-pancreatic fat stranding (arrowhead). The patient presented with interstitial oedematous pancreatitis. Two months later, axial contrastenhanced MDCT (b) shows absent parenchymal enhancement of the pancreatic graft (white asterisk) and intra-pancreatic gas bubbles (white arrowhead). These findings were consistent with necrotising pancreatitis and graft pancreatectomy was performed. Black asterisk renal graft

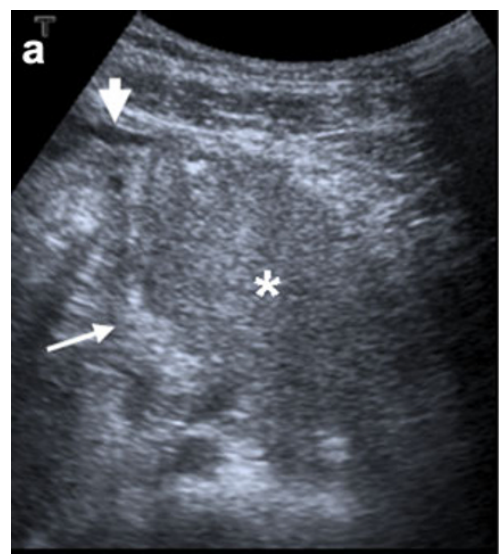

Fig. 7 A 36-year-old woman after SPK transplantation presenting graft pancreatitis. a Ultrasound shows enlarged pancreatic graft (asterisk) with adjacent hyperechoic fatty tissue (thin arrow) and a small amount of free intraperitoneal fluid (large arrow). b Axial

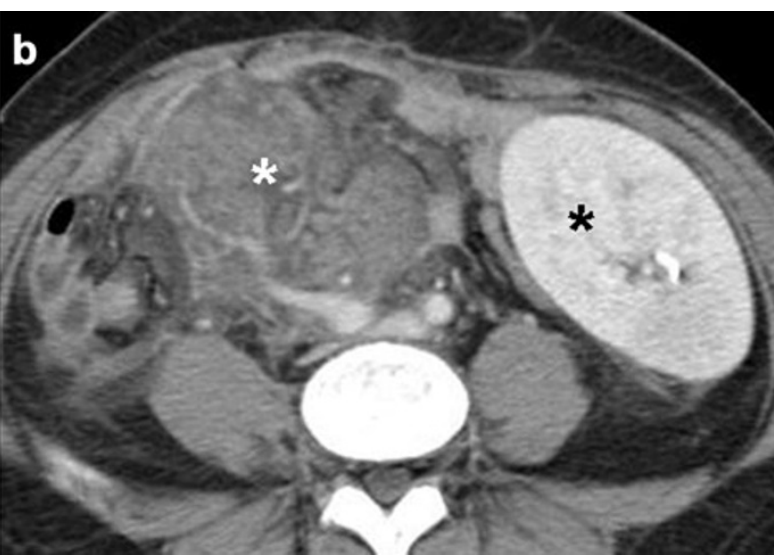

contrast-enhanced helical CT shows an enlarged pancreatic graft with heterogeneous contrast enhancement (white asterisk), peri-pancreatic fat stranding and free peritoneal fluid. Normal renal graft is located on the left (black asterisk) 


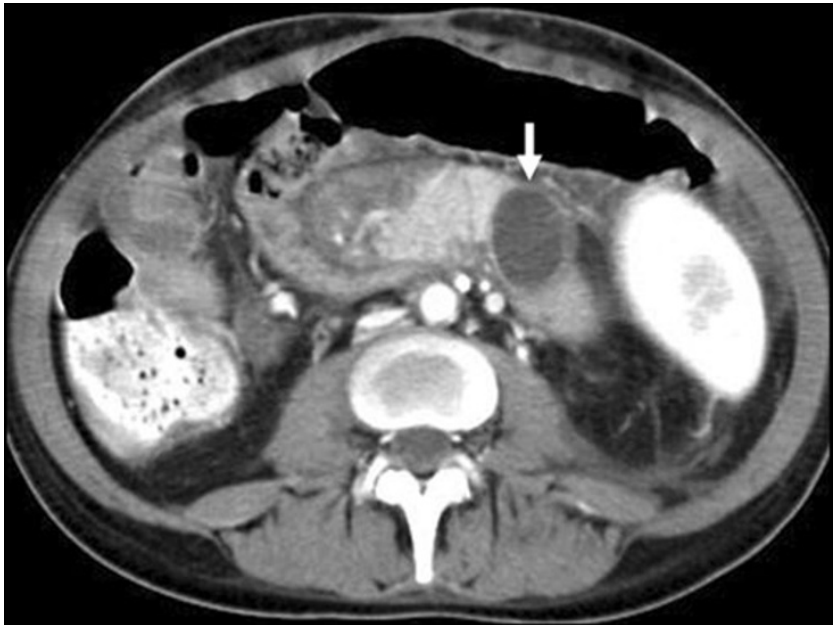

Fig. 9 A 36-year-old male patient after SPK transplantation with pancreatitis and pseudocyst formation. Axial contrast-enhanced CT shows heterogeneous enhancement of the pancreatic graft and a thinwalled pseudocyst (arrow)
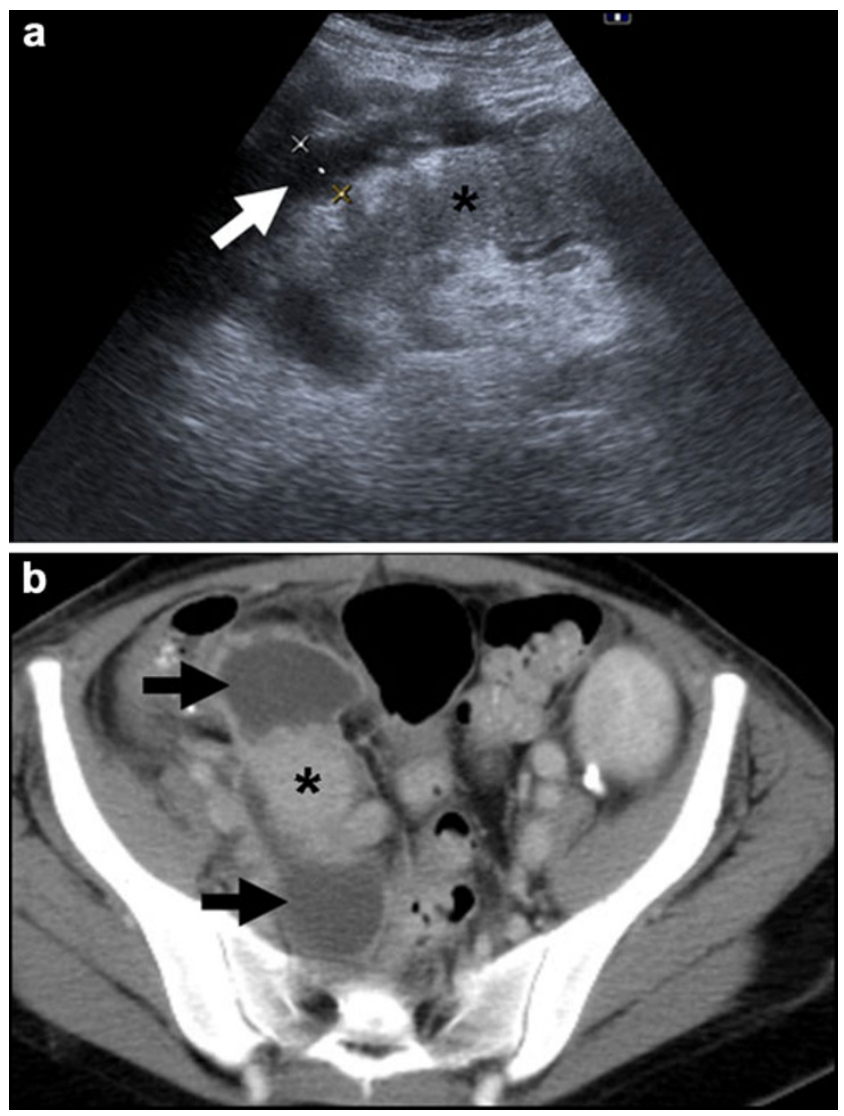

Fig. 10 A 30-year-old woman 5 weeks after SPK transplantation with an infected peri-pancreatic pseudocyst. Sagittal ultrasound of the right lower quadrant (a) demonstrates a peri-pancreatic fluid collection (white arrow). Axial contrast-enhanced MDCT (b) shows homogeneous contrast enhancement of the pancreatic graft (asterisk) surrounded by a thin, contrast-enhanced wall fluid collection (black arrows). Image-guided percutaneous drainage confirmed superimposed infection of the pseudocyst heterogeneous parenchymal enhancement. As imaging and laboratory values are non-specific, graft biopsy is the only reliable test to diagnose graft rejection [10]. Graft biopsy can be performed under ultrasound or CT guidance (Fig. 11), and a low rate of complications has been previously reported [11].

\section{Fistulous tracts}

A pancreatic fistula usually appears as peri-pancreatic graft collection possessing high amylase levels when a puncture is performed. On MRCP, communication with the main duct may be identified. Although fistulas tend to resolve with conservative treatment, infection (abscess) or fistulous tract to the skin, peritoneal cavity, gut or uterine cavity may also develop; thus, follow-up imaging should be proposed (Fig. 12) [8].

\section{Post-transplant lymphoproliferative disease}

Lymphoproliferative disease is a rare late complication and has been reported with an incidence of 3-12\% after pancreatic transplantation [5, 12]. In our institution, no cases have been diagnosed so far. Imaging may depict diffuse graft enlargement, indistinguishable from acute pancreatitis or rejection, but typically unresponsive to immunosuppressive therapy. Focal masses, inside or outside the graft, lymphadenopathy and/or organomegaly may also be seen [12].

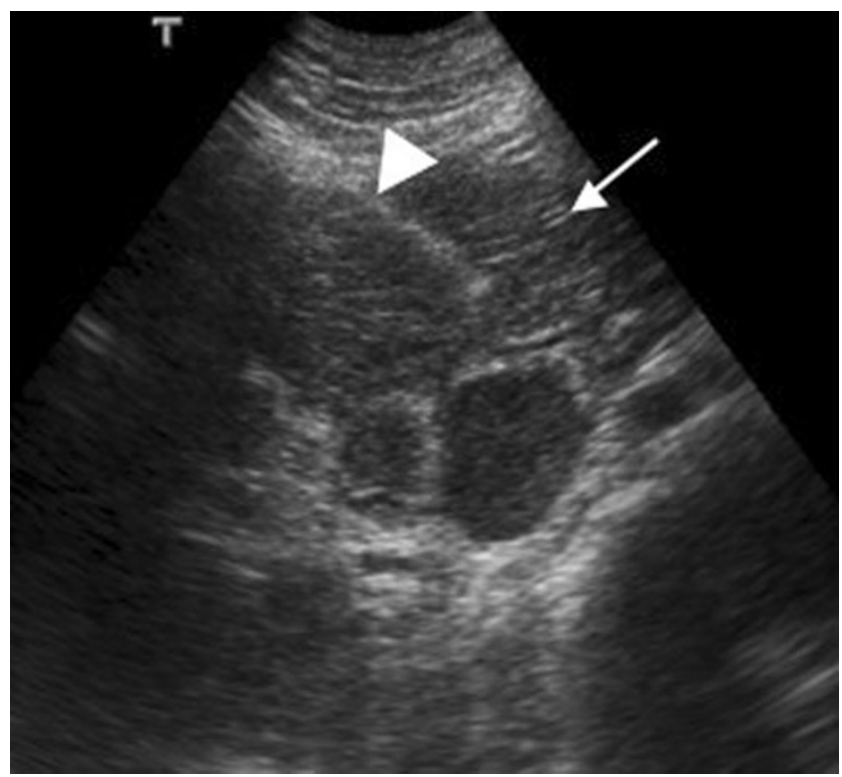

Fig. 11 A 29-year-old patient with rejection of the pancreatic graft after SPK transplantation. Ultrasound-guided biopsy of the pancreatic graft (arrowhead needle biopsy). Pancreatic graft (arrow) shows normal size but with heterogeneous texture of the parenchyma 

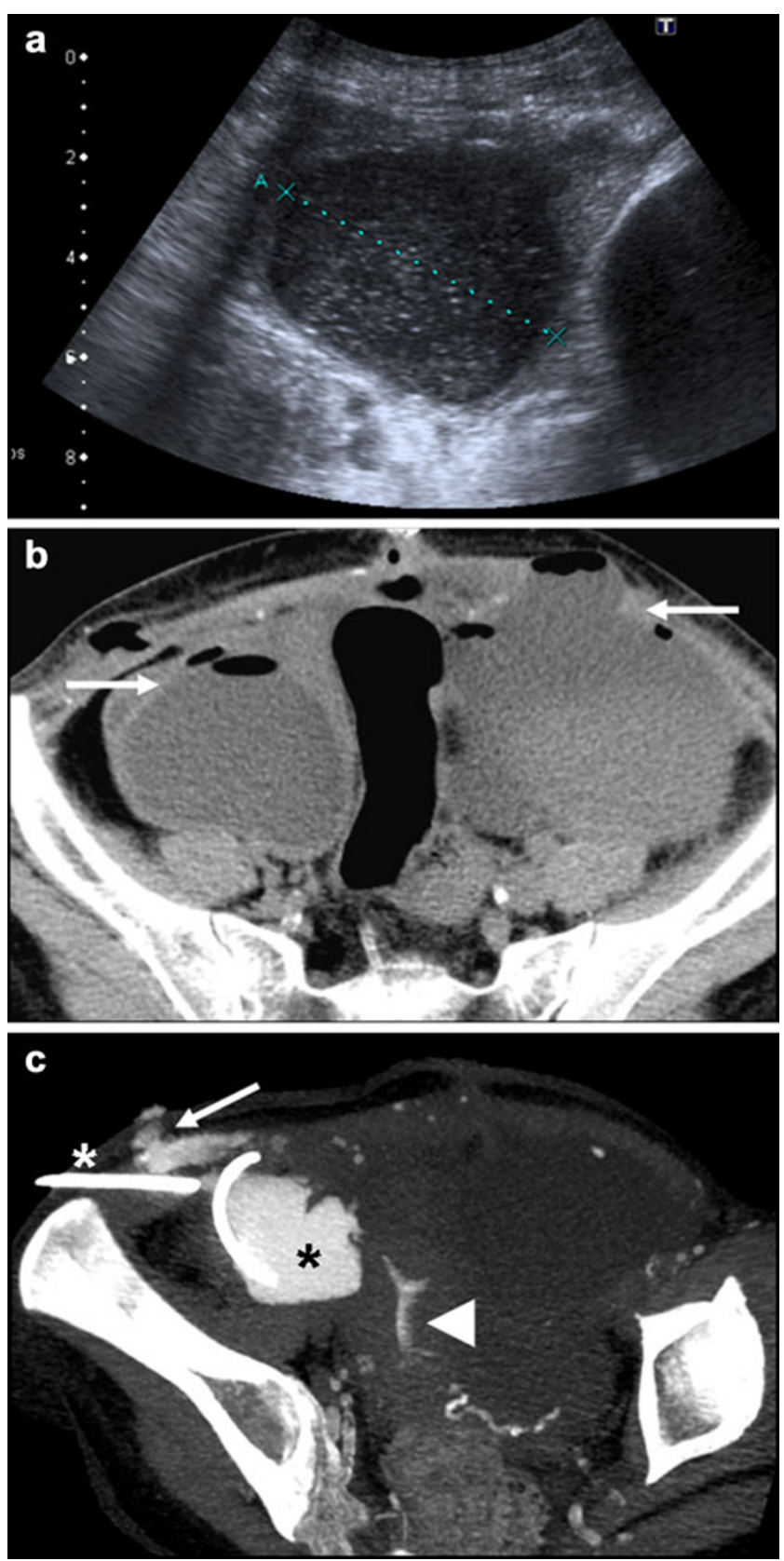

Fig. 12 A 30-year-old woman after SPK transplantation. a Abdominal ultrasound shows peri-pancreatic graft collections with a thick wall and echogenic content; $\mathbf{b}$ axial MDCT reveals multiple abscesses at the abdomen and pelvis, with extension to the abdominal wall. Ultrasound-percutaneous drainage was performed and chemical analysis of the fluid showed high amylase content, compatible with a pancreatic fistula. c A 3D-MPR MIP reconstructed from follow-up MDCT performed 3 days later, with introduction of iodinated contrast agent through the percutaneous drain (white asterisk), demonstrates the extension of intra-abdominal collection (black asterisk) and the fistulous tract to the abdominal wall (arrow). Contrast agent was also detected inside the uterine cavity (arrowhead) suggesting a fistula. Despite this complication, the pancreatic and renal grafts functioned normally
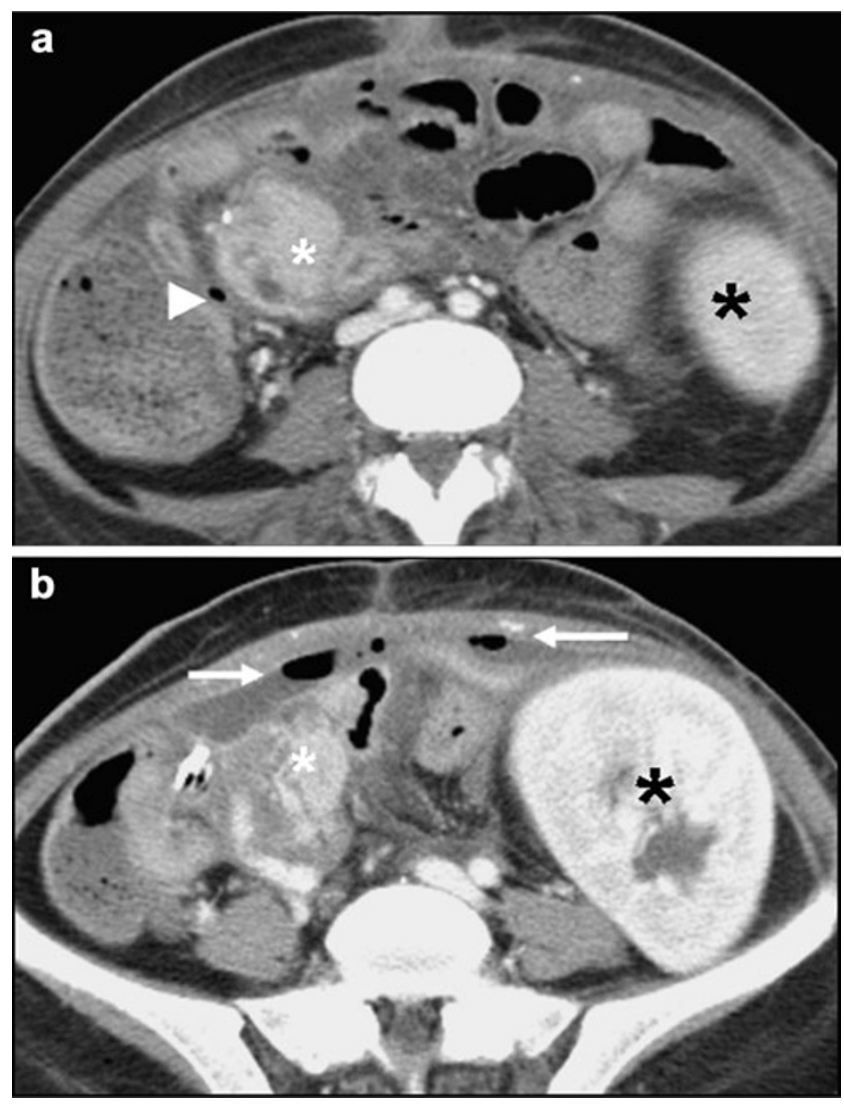

Fig. 13a, b A 30-year-old woman after SPK transplantation. Axial MDCT performed six weeks after transplantation shows intraabdominal fluid collections with contrast-enhancing wall, with airfluid levels (arrows), consistent with abscesses. Ascites and gas bubbles (arrowhead) are noted near the duodeno-enterostomy. The pancreatic (white asterisk) and renal grafts (black asterisk) enhance homogeneously. Duodenal dehiscence was suggested. The patient underwent surgery, which revealed a fistula at the donor's duodenal cuff

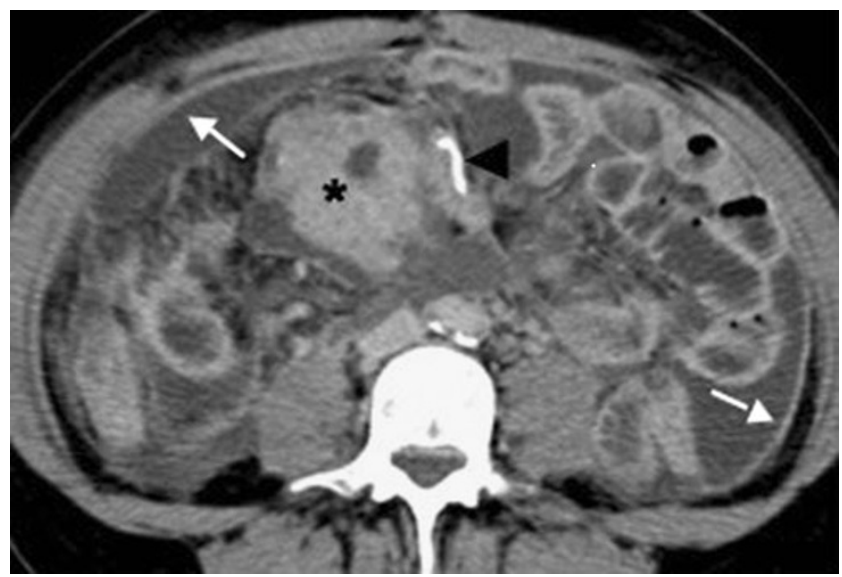

Fig. 14 A 37-year-old woman with peritonitis 5 weeks after SPK transplantation. Contrast-enhanced MDCT shows fluid collections surrounded by enhanced peritoneum (arrows). Asterisk pancreatic graft, arrowhead surgical staples at the graft's duodenal segment 


\section{Infection}

Abscesses may result from infection of peri-pancreatic fluid collections, pseudocysts (Fig. 10), leakage of the enteric anastomosis (Fig. 13), or abdominal wall surgical wound infection [5]. They usually present as complex fluid collections, with a thick wall and possible intralesional gas. Either ultrasound or CT can be used to guide the percutaneous drainage of these collections.

\section{Enteric complications}

These are mainly represented by anastomotic leakage at the duodeno-enterostomy site and small bowel obstruction. CT is especially useful for evaluating these complications, directly demonstrating intra-abdominal abscesses, peritoneal inflammation (Figs. 13 and 14), or the enteric leakage observed as
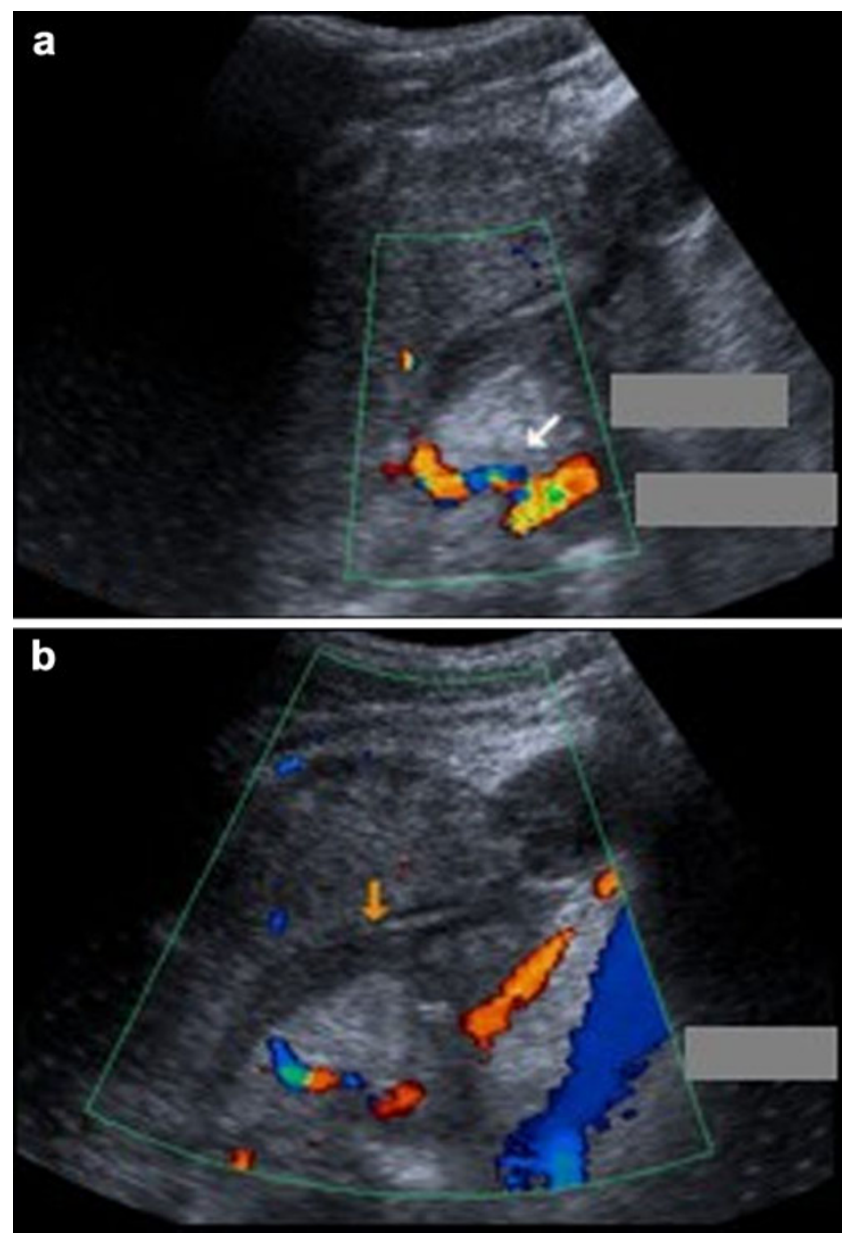

Fig. 15a, b A 37-year-old man after SPK transplantation, with persistent amylase elevation. Abdominal ultrasound-Doppler performed 2 weeks after transplantation shows the iliac arteries and the graft's arterial vessels (a arrow) permeable but with aliasing and high resistive index (RI). The iliac vein showed normal flow. However, no flow was detected at the graft's portal vein, suggesting thrombosis (b arrow). At surgery, thrombosis was confirmed and pancreatectomy was performed extravasation of orally administered contrast agent. The most common cause of small bowel obstruction after abdominal surgery is intestinal adhesions, but obstructions due to internal hernias or volvulus have also been reported [13]. Colonic infections, such as CMV infection, Clostridium difficile colitis or typhlitis, may occur, related to antibiotic therapy and to the immunocompromised state of the patient. These conditions may be suspected whenever colonic wall thickening with increased contrast enhancement is observed [7].

Vascular complications

Venous or arterial graft thrombosis is a serious complication, being the second most common cause of transplant dysfunction after graft rejection [14]. Generally, it results in massive graft necrosis and requires pancreatectomy [8]. Doppler ultrasound depicts parenchymal heterogeneity with absent pulsatile or continuous flow of the pancreatic graft vessels (Fig. 15). In cases of venous thrombosis, pan-diastolic reversal of the arterial flow and a resistive index greater than 1.0 can be seen [9]. Intravenous contrast agent administration is especially useful for demonstrating intraluminal filling defects in the graft vessels and the lack of the parenchyma's enhancement (Fig. 16) [8, 15-17]. Emphysematous changes can occur with further progression to parenchymal necrosis. When superimposed infection is suspected, image-guided biopsy should be performed [8].

Early arterial occlusion of the pancreatic graft is usually due to technical surgical difficulties involved in performing the ligation of small pancreatic arterial vessels during organ harvesting. Late occlusion is generally related to the end point of graft rejection because of progression of the alloimmune response [8].

Arterial or venous pseudoaneurysms may develop after pseudocyst infection or after biopsy $[8,9,18]$. On Doppler ultrasound, they present as blood-filled lesions. Direct communication with the feeding vessel may also be identified and pulsed-wave Doppler ultrasound may show "to-and-fro" waveform at the pseudoaneurysm neck. Contrast-enhanced CT or MRI is better for demonstrating the focal loss of vessel wall integrity (Fig. 17).

When a simultaneous pancreas-kidney transplant is performed, with bilateral revascularisation to the respective iliac vessels, delayed opacification of the iliac vein ipsilateral to the pancreatic graft may be observed, compared with the contralateral iliac vein draining the kidney transplant (Fig. 18) [19]. This finding should not be interpreted as a real thrombosis of the iliac vein.

Although acute bleeding is rare, it may appear during the early postoperative period and is usually clinically suspected. Ultrasound demonstrates fluid collections but CT better identifies haemorrhage because of its spontaneous hyperdensity on non-enhanced images. After intravenous contrast 
Fig. 16a-d A 37-year-old patient, the same patient as in Fig. 17, a day after a second pancreatic transplant. Axial MDCT (a, b) shows heterogeneous enhancement of the pancreatic head of the graft with normal, contrast-enhancing iliac arteries and the graft's splenic artery (arrow). Poor enhancement of the graft's SMA is noted (arrowhead), together with nonenhancing arterial segments, suggesting occlusion. There is homogeneous enhancement of the renal graft (black asterisk). Coronal reformatted MIP image (c) and VR reconstruction (d) illustrate the graft's splenic artery (arrow) but not the graft's SMA. The patient underwent pancreatectomy and the arterial occlusion was confirmed
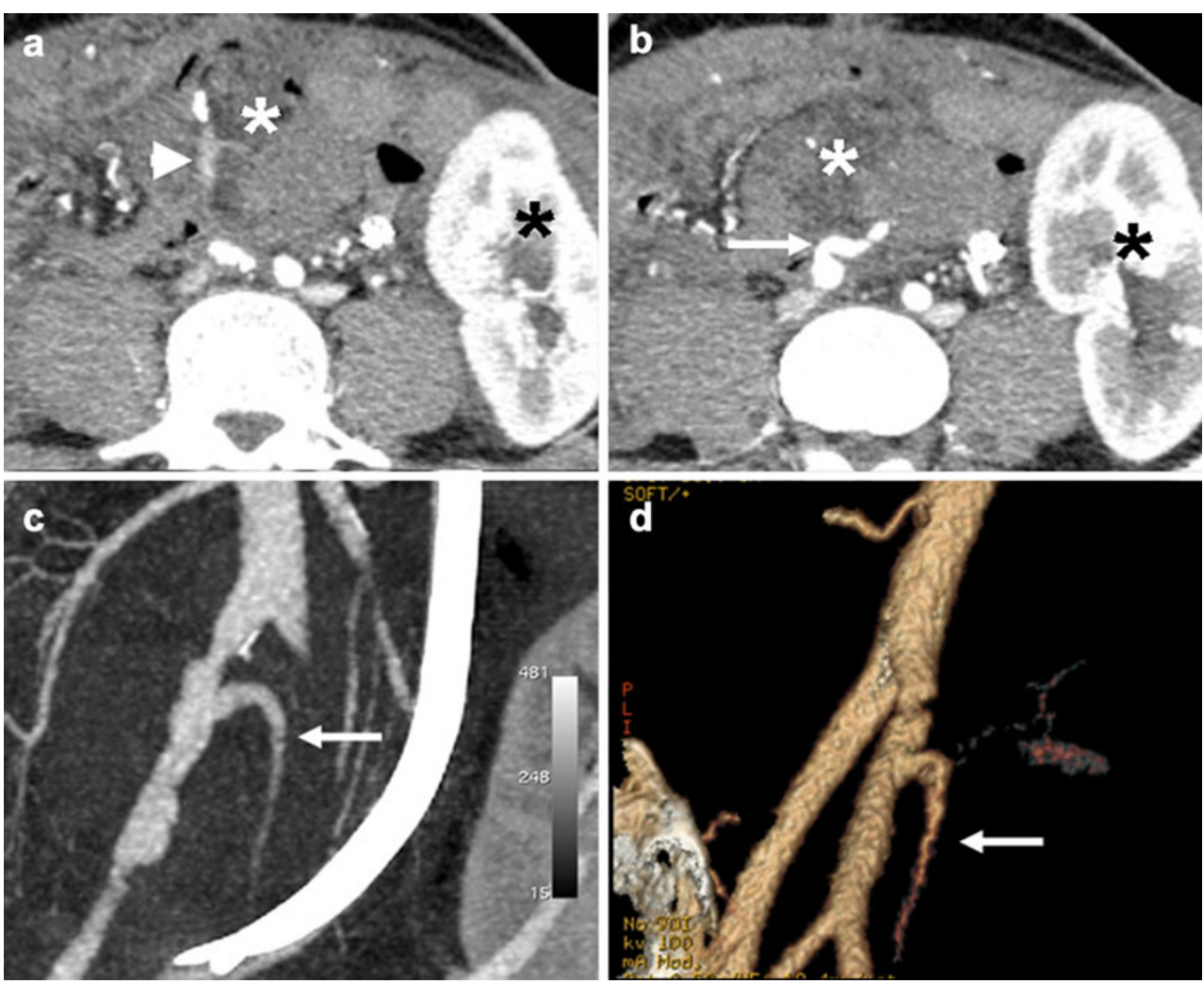

medium administration, extravasation of the contrast medium may be seen and the bleeding point identified [8, 9].

\section{Conclusion}

Different imaging techniques can assess postoperative pancreatic graft. Although Doppler ultrasound is the first-

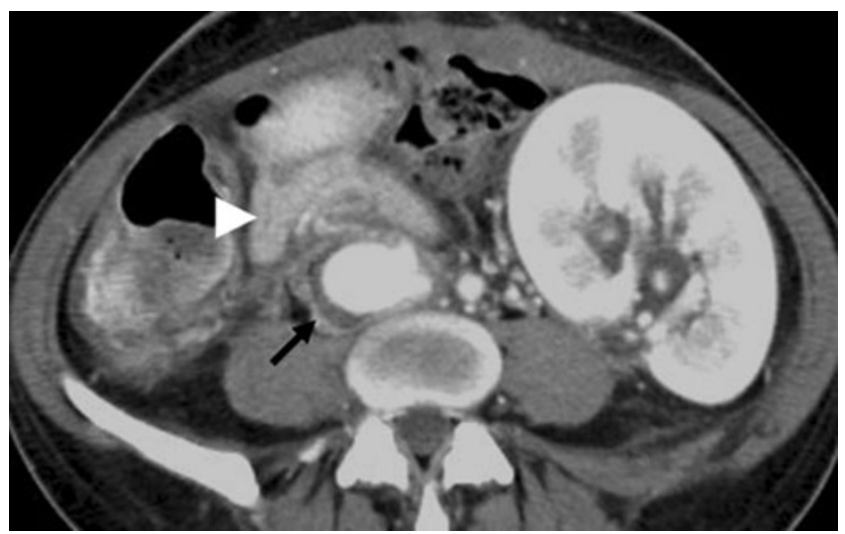

Fig. 17 A 36-year-old female patient after SPK transplantation. Contrast-enhanced CT performed 4 weeks after graft pancreatitis shows homogeneous pancreatic graft enhancement (arrowhead) and a partially thrombosed pseudoaneurysm of the right common iliac artery (arrow) line technique, $\mathrm{CT}$ and, to a minor degree, MRI, have been increasingly performed when ultrasound findings are equivocal.

Radiologists should know the imaging appearances of normal pancreatic grafts, be able to recognise early and late complications related to this complex surgical procedure, contribute to the clinical management and, ultimately, to the long-term survival of pancreatic grafts.

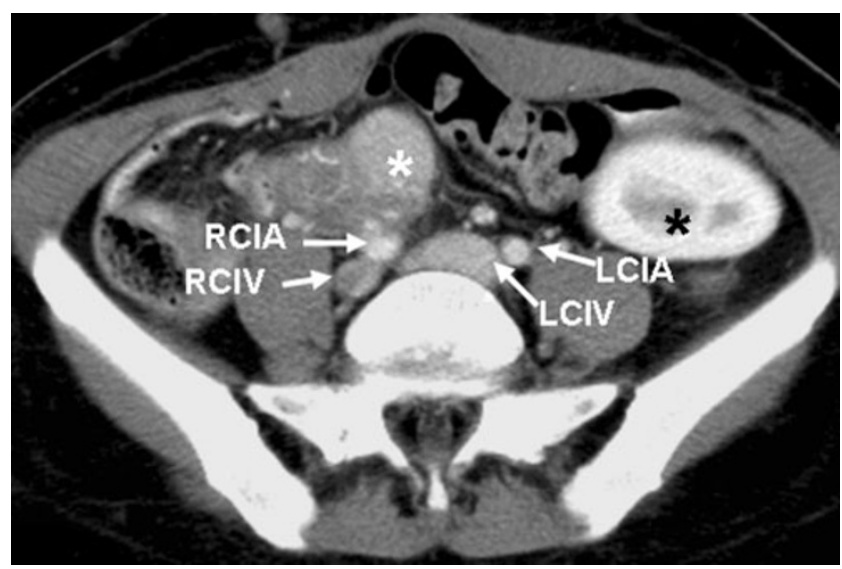

Fig. 18 A 44-year-old woman after SPK transplantation. Contrastenhanced MDCT shows non-enhancement of the right common iliac vein compared with left side. This results from the longer transit time and the reduced blood flow of the right-sided pancreatic graft (white asterisk) compared with the left-sided renal graft (black asterisk) 


\section{References}

1. Leichtman AB, Cohen D, Keith D et al (2008) Kidney and pancreas transplantation in the United States, 1997-2006: the HRSA Breakthrough Collaboratives and the 58 DSA Challenge. Am J Transplant 8:946-957

2. Kelly WD, Lillehei RC, Merkel FK, Idezuki Y, Goetz FC (1967) Allotransplantation of the pancreas and duodenum along with the kidney in diabetic nephropathy. Surgery 61:827-837

3. Freund MC, Steurer W, Gassner EM et al (2004) Spectrum of imaging findings after pancreas transplantation with enteric exocrine drainage: part 1, posttransplantation anatomy. AJR Am J Roentgenol 182:911-917

4. Nikolaidis P, Amin RS, Hwang CM et al (2003) Role of sonography in pancreatic transplantation. Radiographics 23:939-949

5. Sandrasegaran K, Lall C, Berry WA, Hameed T, Maglinte DD (2006) Enteric drainage pancreas transplantation. Abdom Imaging 31(5):588-595

6. Aideyan OA, Foshager MC, Benedetti E, Troppmann C, Gruessner RW (1997) Correlation of the arterial resistive index in pancreas transplants of patients with transplant rejection. AJR Am J Roentgenol 168:1445-1447

7. Dachman AH, Newmark GM, Thistlethwaite JR Jr, Oto A, Bruce DS, Newell KA (1998) Imaging of pancreatic transplantation using portal venous and enteric exocrine drainage. AJR Am J Roentgenol 171:157-163

8. Freund MC, Steurer W, Gassner EM et al (2004) Spectrum of imaging findings after pancreas transplantation with enteric exocrine drainage: part 2, posttransplantation complications. AJR Am J Roentgenol 182:919-925

9. Dillman JR, Elsayes KM, Platt JF, Bude RO, Francis IR (2009) Imaging of pancreas transplants: postoperative findings with clinical correlation. J Comput Assist Tomogr 33(4):609-617
10. Lee BC, McGahan JP, Perez RV, Boone JM (2000) The role of percutaneous biopsy in detection of pancreatic transplant rejection. Clin Transplant 14:493-498

11. Atwell TD, Gorman B, Larson TS, Charboneau JW, Ingalls Hanson BM, Stegall MD (2004) Pancreas transplants: experience with 232 percutaneous US-guided biopsy procedures in 88 patients. Radiology 231:845-849

12. Meador TL, Krebs TL, Cheong JJ, Daly B, Keay S, Bartlett S (2000) Imaging features of posttransplantation lymphoproliferative disorder in pancreas transplant recipients. AJR Am J Roentgenol 174(1):121-124

13. Lall CG, Sandrasegaran K, Maglinte DT, Fridell JA (2006) Bowel complications seen on CT after pancreas transplantation with enteric drainage. AJR Am J Roentgenol 187:1288-1295

14. Chandra J, Phillips RR, Boardman P, Gleeson FV, Anderson EM (2009) Pancreas transplants. Clin Radiol 64:714-723

15. Hagspiel KD, Nandalur K, Burkholder B et al (2005) Contrastenhanced MR angiography after pancreas transplantation: normal appearance and vascular complications. AJR Am J Roentgenol 184:465-473

16. Hagspiel KD, Nandalur K, Pruett TL et al (2007) Evaluation of vascular complications of pancreas transplantation with highspatial-resolution contrast-enhanced MR angiography. Radiology 242:590-599

17. Dobos N, Roberts DA, Insko EK, Siegelman ES, Naji A, Markmann JF (2005) Contrast-enhanced MR angiography for evaluation of vascular complications of the pancreatic transplant. Radiographics 25:687-695

18. Madan AK, Allmon JC, Harding M, Cheng SS, Slakey DP (2001) Extrapancreatic pseudoaneurysm after pancreas transplantation. Transplantation 71:1853-1856

19. Gupta R, Rottenberg G, Taylor J (2002) Pseudothrombosis of the iliac vein in patients following combined kidney and pancreas transplantation. Br J Radiol 75:692-694 